\title{
Endoscopic Gallbladder Drainage in Medically Inoperable Patients with Symptomatic Cholelithiasis: A Tube to Avoid "Going Down the Tubes"?
}

\author{
Raj J. Shah ${ }^{1,2}$
}

Published online: 29 May 2015

(c) Springer Science+Business Media New York 2015

Gastrointestinal (GI) practitioners and trainees are often faced with an elderly curmudgeon sitting in the front row of GI grand rounds with the primary aim of putting a "hole" into the interventional endoscopist's data promoting the latest endoscopic innovation. The comments made by these "elder statesmen" are at times appropriate regarding the importance of "evidenced-based" research, i.e., data obtained from prospective, randomized controlled trials. However, if this standard was needed prior to adoption of procedures, there would have been little for interventional gastroenterologists to offer to patients with pancreaticobiliary diseases over the last 20 years other than to remove bile duct stones or to sample pancreatic masses. The lack of prospective, randomized trials to support new endoscopic therapies should thus not temper enthusiasm for novel therapies that may improve patients' lives.

Such a non-"evidence based" approach has been applied to stenting the gallbladder, a far-from-simple procedure indicated for biliary drainage and gallbladder decompression in patients with symptomatic cholelithiasis who are medically inoperable. The formerly prevailing procedure, placement of percutaneous cholecystostomy tubes, is fraught with complications (e.g., drain dislodgement and occlusion, leak, infection, bleeding, and discomfort) although placement can be lifesaving in the medically unfit patient $[1,2]$. In one study of 68 patients, success was noted in $85 \%$

Raj J. Shah

raj.shah@ucdenver.edu

1 Department of Medicine, University of Colorado School of Medicine, Aurora, CO, USA

2 University of Colorado Anschutz Medical Campus, 1635 Aurora Ct, Mail Stop F735, Rm. AIP 2.031, Aurora, CO 80045, USA although complications occurred in $16 \%$ [2]. Furthermore, a high percentage $(41 \%)$ of patients who did not subsequently undergo cholecystectomy developed recurrent gallbladder-related symptoms.

Although endoscopic cystic duct stenting is a simplesounding procedure, it can be technically challenging. For example, the cystic duct takeoff may be acute and difficult to identify or selectively cannulate, further complicated by a tortuous course, narrow caliber, and the valves of Heister. Therefore, as with any therapeutic endoscopic intervention, informed patient selection is essential to avoid undue harm, identifying those who are most likely to gain long-term benefit.

In this issue of Digestive Diseases and Sciences, McCarthy et al. [3] retrospectively identified 29 patients without cirrhosis over a 9-year period who underwent gallbladder stenting for symptomatic cholelithiasis. Fully $86 \%$ of the patients were medically inoperable due to comorbidities that included cardiopulmonary disease and an American Society of Anesthesiology's (ASA) classification of III/IV. The study cohort underwent endoscopic retrograde cholangiopancreatography (ERCP) with 5-7F double pigtail stents placed into the gallbladder. Technical success was noted in $62 \%$ after a single attempt and in $76 \%$ after two attempts; $80 \%$ had biliary sphincterotomy. No reported peri-procedural morbidity occurred though two patients developed the delayed complications of abdominal pain and cholangitis. The rate of technical success was not optimal despite six patients who had percutaneous "rendezvous" procedures to gain endoscopic gallbladder access. Failure was noted in those with complete obstruction of the cystic duct, ironically the same group who would seemingly have benefited the most from a decompressive intervention. Nevertheless, clinical improvement was noted in $90 \%$ at 1 year. Stents were changed only as 
needed; $21 \%$ of the cohort had 21/2 years of follow-up with resolution of symptoms without stent exchange. Yet, nearly one-quarter of the subjects had gallbladder stenting for choledochal problems that could likely have been treated with sphincterotomy alone [4]. On follow-up, 5/29 (17\%) underwent cholecystectomy; the gallbladder stenting intervention may thus have served as a useful "bridge" to cholecystectomy until comorbidities were optimized.

As the authors note and as would stand to reason, transpapillary gallbladder stents may relieve symptoms of biliary colic via one or more of the following mechanisms: (1) facilitating bile drainage via a "wicking" mechanism that does not require stent patency, (2) decompressing the gallbladder and preventing refilling, thereby preventing distention and ischemia of the gallbladder wall, and (3) preventing cystic duct impaction with a stone. Thus, it is conceivable that patients with biliary colic but patent cystic ducts might receive benefit from endoscopic sphincterotomy alone to promote transpapillary bile drainage.

Stenting of the gallbladder was first described in the early 1990s [5, 6]. In a small retrospective series of 23 patients over a 10-year period, our group evaluated its efficacy in cirrhotic patients with biliary colic awaiting liver transplantation [7]. Technical success was achieved in all patients, stents were changed on an as-needed basis, and there were no acute complications. The limitations are that it is a technically demanding procedure if the takeoff of the cystic duct orifice is not readily apparent. Complications of ERCP with gallbladder stenting are probably underreported in the literature as well; for example, I have encountered cystic duct perforation during attempted wire placement and dilation of a cystic duct stricture. This resolved after bile duct stent insertion and subsequently, gallbladder stenting was successful.

An additional challenge is when the cystic duct is completely obstructed, rendering transpapillary access nearly impossible. In this circumstance, the gallbladder may be drained via the gastric antrum or duodenal bulb using endoscopic ultrasound [8,9]. Because the gallbladder is not adherent to the luminal gastrointestinal tract, fully covered self-expandable metallic stents (SEMS) have facilitated organ apposition for drainage. In one series of 63 patients, technical and clinical success was reported in $98 \%$ of patients [8]. Complications of duodenal perforation $(n=1)$ and pneumoperitoneum $(n=2)$ resolved with conservative management. Late adverse events included distal stent migration $(n=2)$ and acute cholecystitis due to stent occlusion $(n=2)$, both treated with endoscopic re-intervention. The cumulative stent patency rate was $86 \%$ at 3 years. The development of a novel lumen-apposing fully covered metal stent may permit anchoring and be ideal for transenteric gallbladder drainage [9].

In institutions that provide liver transplantation services, preoperative gallbladder stenting has proven useful, since the risk from cholecystectomy is prohibitive due to comorbidities such as coagulopathy or thrombocytopenia. Gallbladder stenting has consequently revolutionized the management of symptomatic cholelithiasis in this population.

If symptoms are related to cystic duct obstruction, it seems logical, even without prospective randomized trials, that endoscopic decompression improves symptoms. Although prospective randomized studies would be welcomed, this therapy should not be withheld pending completion of those studies. After all, is a randomized prospective trial needed to know that parachutes save the lives of individuals who jump out of airplanes? [10].

\section{References}

1. Anderson JE, Chang DC, Talamini MA. A nationwide examination of outcomes of percutaneous cholecystostomy compared with cholecystectomy for acute cholecystitis, 1998-2010. Surg Endosc. 2013;27:3406-3411.

2. McKay A, Abulfaraj M, Lipschitz J. Short- and long-term outcomes following percutaneous cholecystostomy for acute cholecystitis in high-risk patients. Surg Endosc. 2012;26:1343-1351.

3. McCarthy S, Tujios S, Fontana R, et al. Endoscopic transpapillary gallbladder stent placement is safe and effective in high-risk patients without cirrhosis. Dig Dis Sci. (Epub ahead of print). doi:10.1007/s10620-014-3371-4.

4. Siegel JH, Veerappan A, Cohen S, et al. Endoscopic sphincterotomy for biliary pancreatitis: an alternative to cholecystectomy in high-risk patients. Gastrointest Endosc. 1994;40:573-575.

5. Tamada K, Seki H, Sato K, et al. Efficacy of endoscopic retrograde cholecystoendoprosthesis (ERCCE) for cholecystitis. Endoscopy. 1991;23:1-2.

6. Kalloo AN, Thuluvath PJ, Pasricha PJ. Treatment of high-risk patients with symptomatic cholelithiasis by endoscopic gallbladder stenting. Gastrointest Endosc. 1994;40:608-610.

7. Schlenker C, Trotter JF, Shah RJ, et al. Endoscopic gallbladder stent placement for treatment of symptomatic cholelithiasis in patients with end-stage liver disease. Am J Gastroenterol. 2006;101:278-283.

8. Choi JH, Lee SS, Choi JH, et al. Long-term outcomes after endoscopic ultrasonography-guided gallbladder drainage for acute cholecystitis. Endoscopy. 2014;46:656-661.

9. Itoi T, Binmoeller KF, Shah J, et al. Clinical evaluation of a novel lumen-apposing metal stent for endosonography-guided pancreatic pseudocyst and gallbladder drainage (with videos). Gastrointest Endosc. 2012;75:870-876.

10. Gordon C, Smith S, Pell J. Parachute use to prevent death and major trauma related to gravitational challenge: systematic review of randomised controlled trials. BMJ. 2003;327:1459-1460. 